\begin{tabular}{lllll}
\hline Estudios de Economía Aplicada & Vol. 30-2 2012 & PÁgs. 637-654 \\
\hline
\end{tabular}

\title{
Los avances en la valoración económica del deporte en Europa
}

\author{
JOSÉ J. BENÍTEZ ROCHEL \\ Departamento de Economía Aplicada (Política Económica), UNIVERSIDAD DE MÁLAGA, \\ ESPAÑA.E-mail: jjbenitez@uma.es \\ BEATRIZ LACOMBA ARIAS \\ Departamento de Economía Aplicada (Estadística y Econometría), UNIVERSIDAD DE \\ MÁLAGA,ESPAÑN.E-mail: beatriz@uma.es
}

\section{RESUMEN}

Este trabajo se centra en los avances que se han producido en Europa en la valoración económica del deporte a nivel macroeconómico. En primer lugar, se analizan los primeros intentos por estimar la importancia económica del deporte en Europa y que pusieron de manifiesto la necesidad de utilizar unas definiciones y metodologías comunes. Sobre esa base, se discuten los progresos que se han producido en este ámbito. A continuación se hace una especial referencia al caso español, incluyendo una aproximación tentativa al peso económico del sector.

Palabras clave: Deporte, Europa, valoración.

\section{Advances in Economic Valuation of Sport in Europe}

\begin{abstract}
This work focuses on the advances that have occurred in Europe in the economic valuation of sport at the macroeconomic level. First of all, we analyze the attempts to estimate the economic importance of sport in Europe and highlighted the need for common definitions and methodologies. On that basis, we discuss the progress that has been produced in this area. The following is a special reference to Spain, including a tentative approach to the economic weight of sport.
\end{abstract}

Keywords: Sport, Europe, Valuation.

Clasificación JEL: L83

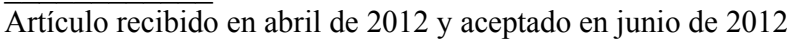

Artículo disponible en versión electrónica en la página www.revista-eea.net, ref. ə-30214 


\section{INTRODUCCIÓN}

El estudio del deporte puede realizarse desde muy diferentes enfoques. Incluso, desde el punto de vista económico, admite distintas aproximaciones. En Estados Unidos, desde la seminal aportación de Rottenberg (1956) que inició, para algunos, la denominada "economía del deporte" (Noll, 2006), la investigación se ha orientado, fundamentalmente, hacia el análisis del deporte profesional y ha alcanzado un elevado grado de rigurosidad. Por ejemplo, desde 2000, se edita el Journal of Sports Economics que recoge, de acuerdo con los estándares convencionales de calidad científica, buena parte de las investigaciones sobre el tema y que se presenta como "la única publicación dedicada específicamente a este campo". En Europa, en cambio, las contribuciones han sido menos numerosas y, en muchos casos, se han orientado hacia el impacto económico del deporte distinguiéndose aquellas que, a nivel microeconómico, tratan de evaluar las consecuencias de, por ejemplo, la celebración de un evento deportivo o de la construcción de una determinada instalación deportiva, de aquellas otras que se ocupan de medir la importancia del deporte en el conjunto de la economía. Precisamente, este trabajo se centra en los avances que se han producido en Europa en la valoración económica del deporte a nivel macroeconómico.

En primer lugar, analizaremos los primeros intentos por estimar la importancia económica del deporte en Europa y que, como trataremos de argumentar, pusieron de manifiesto la necesidad de disponer de unos conceptos y metodologías comunes. Sobre esa base, en los siguientes epígrafes discutiremos los progresos que se han producido en este ámbito y los primeros resultados obtenidos. A continuación haremos una especial referencia al caso español. Terminaremos con un apartado de conclusiones.

\section{ANTECEDENTES}

La primera referencia sobre la importancia económica del deporte a nivel europeo se encuentra en Jones (1989). El estudio se llevó a cabo a iniciativa del Comité para el Desarrollo del Deporte del Consejo de Europa y aportó información sobre nueve países: Bélgica, Dinamarca, Finlandia, Francia, Holanda, Islandia, Portugal, República Federal de Alemania y Reino Unido. A pesar de sus evidentes limitaciones metodológicas, proporcionó las primeras estimaciones comparables sobre el porcentaje que representaba el deporte en el PIB. Los datos demostraron que, dentro de la diversidad de situaciones, la actividad económica que generaba el deporte era relevante. En 1985, el porcentaje que representaba sobre el PIB oscilaba entre el $0.9 \%$ de Finlandia y el $1.8 \%$ de Holanda.

La segunda referencia, también auspiciada por el Consejo de Europa, es la de Andreff et alia (1994). Su objetivo era más ambicioso que el del anterior documento puesto que no se limitaba a recopilar información ya existente sino 
que aspiraba a aportar nuevos datos a través de un cuestionario que permitiera disponer de un marco contable normalizado en doce países; se añadió España, Grecia, Hungría, Italia y Suiza y se eliminó, respecto a la investigación de Jones (1989), a Italia y a Holanda. Aunque los problemas metodológicos no desaparecieron por completo y los objetivos no se alcanzaron plenamente, los resultados obtenidos confirmaron, en términos generales, tanto la importancia económica del deporte como la diversidad de situaciones en los países analizados. En 1990, y para los siete países comunes con el estudio anterior, el porcentaje que alcanzaba el deporte sobre PIB variaba desde el $0.6 \%$ de Dinamarca al $1.8 \%$ de Portugal. Si se consideraban todos los países destacaba el caso de Suiza que llegaba a suponer un $3.5 \%$ sobre el PIB.

Problemas financieros impidieron que se continuara esta línea de investigación abierta en el seno del Consejo de Europa. No obstante, posteriormente, surgieron distintas iniciativas que, a nivel nacional, estaban aportando datos que cuantificaban el peso del deporte en las principales macromagnitudes. En la Tabla 1 se recogen algunas referencias.

Tabla 1

Estudios sobre la incidencia económica del deporte en Europa

\begin{tabular}{|l|l|c|c|}
\hline \multicolumn{1}{|c|}{ Autores } & \multicolumn{1}{c|}{ País } & PIB (\%) & Año \\
\hline Mussino (1994) & Italia & 2.4 & 1996 \\
\hline Taks y Késenne (2000) & Flandes & 3.7 & 1996 \\
\hline Ahlert (2000) & Alemania & 1.4 & 1998 \\
\hline Bednarik, Kolar y Jurak (2010) & Eslovenia & 1.9 & 2005 \\
\hline Ministère de la Santé et des Sports (2009) & Francia & 1.8 & 2007 \\
\hline SIRC (2010) & Gran Bretaña & 1.5 & 2008 \\
\hline
\end{tabular}

Fuente: Elaboración propia.

Podrían citarse, adicionalmente, otros estudios puntuales que aportan información sobre la importación del deporte en el PIB para el conjunto de la Unión Europea. Vocasport (2004) la estimó en $1.6 \%$ y Dimitrov et alia (2006) en un $3.7 \%$ para la UE-25 y en un $4.6 \%$ para la UE-15, entendiendo el deporte en un sentido amplio. Estas diferencias en los resultados es la consecuencia lógica de la aplicación de metodologías y definiciones dispares. De hecho, Andreff (2006) constató que no existía en ningún país una publicación periódica sobre la economía del deporte y que el establecimiento de un sistema contable nacional estaba todavía en ciernes.

En definitiva, a mitad de la década pasada, la información estadística disponible en Europa ponía de manifiesto que, en torno al deporte, se generaba una actividad económica significativa y que, en cualquier caso, se apreciaba una 
diversidad de situaciones en los países analizados. No obstante, aún no existía un consenso sobre cómo habría de abordarse su medición. Sobre esa base, surgieron algunas iniciativas políticas para el desarrollo de una aproximación común a la cuantificación del impacto económico del deporte en la Unión Europea.

En este contexto aparece el Libro Blanco sobre el Deporte de la Comisión de las Comunidades Europeas (2007) en donde se recoge un apartado específicamente dedicado a la dimensión económica. Se reconoce que, aunque su importancia está confirmada por los estudios existentes, y se cita el trabajo de Dimitrov et alia (2006), "no hay datos fiables y comparables sobre el peso económico del deporte" por lo que, aceptada su capacidad para contribuir al crecimiento y la creación de empleo, se afirma que la "Comisión, en estrecha colaboración con los estados miembros, tratará de establecer un método estadístico europeo para medir el impacto económico del deporte como base para las cuentas estadísticas nacionales en la materia, que podría dar lugar, con el tiempo, a una cuenta satélite europea sobre el tema". Aunque, como es evidente, el objetivo se establece con mucha prudencia, lo cierto es que, desde entonces, se han producido algunos avances reseñables.

Las iniciativas, en el ámbito europeo sobre el conocimiento estadístico de la importancia económica del deporte, se empezaron a llevar a cabo a través del grupo de trabajo sobre economía y deporte que había sido creado, previamente, a instancias de la presidencia austriaca de la UE. En su primera reunión, celebrada en septiembre de 2006, se determinó que uno de sus objetivos principales era medir el porcentaje que representaba el "sector deporte" sobre el PIB y sus efectos sobre el empleo, el valor añadido y el poder adquisitivo en los estados miembros así como en el conjunto de la Unión Europea. En las sucesivas reuniones que se celebraron se progresó tanto en la definición económica del deporte como en la metodología para su cuantificación.

\section{DEFINICIÓN DE VILNA}

Uno de los principales obstáculos para cuantificar la aportación al PIB del deporte se encuentra en su propia definición desde el punto de vista económico. El concepto de deporte está sujeto a discusión. Quizás, por ello, aunque las propias constituciones de los países suelen dedicar algunas referencias al deporte e, incluso, es frecuente, como en el caso de España, que se promulguen leyes específicas sobre el deporte, no suele encontrarse una definición en el articulado de estas normas (Soek, 2006). No obstante, en el artículo 2 de la Carta Europea del Deporte, adoptada por el Comité de Ministros del Consejo de Europa se puede leer lo siguiente: "Se entenderá por 'deporte' todo tipo de actividades físicas que, mediante una participación organizada o de otro tipo, tengan por finalidad la expresión o la mejora de la condición física y psíquica, el desarrollo 
de las relaciones sociales o el logro de resultados en competiciones de todos los niveles". Este podría ser un buen punto de partida para la delimitación del "sector deporte" en Europa. El problema es que no se aclara, como, por otro lado, es comprensible, las actividades económicas, ya sea por el lado de la demanda o de la oferta, que habrían de ser consideradas.

Es lógico, por tanto, que uno de los primeros objetivos que se planteó en el seno del grupo de trabajo sobre economía y deporte, que se ha mencionado más arriba, fuera la definición del deporte de una manera que permitiera la recopilación de los datos precisos para su cuantificación desde el punto de vista económico. La cuarta reunión de este grupo se celebró en 2007 en la ciudad lituana de Vilna. Allí se alcanzó un acuerdo sobre la identificación de las distintas categorías de la Nomenclatura Estadística de Actividades Económicas de la Comunidad Europea (NACE) que se correspondería con la definición de deporte.

La "definición de Vilna" constaba, en realidad, de tres de definiciones: la estadística; la reducida y la ampliada. La definición estadística limitaba el deporte a la categoría 92.6 de la NACE Rev. 1 (o la 93.1 de la NACE Rev. 2). Es decir, comprende las "Actividades Deportivas": gestión de instalaciones deportivas; actividades de los clubes deportivos; actividades de los gimnasios; y otras actividades deportivas como, por ejemplo, las que realizan por cuenta propia los deportistas.

La definición reducida, además de incluir a la estadística, se refiere a todos aquellos productos y servicios que son necesarios para la práctica deportiva: calzado, ropa, objetos, infraestructuras, etc.

Por último, la definición ampliada abarca la reducida y le añade todas aquellas categorías de la NACE que usan el deporte como input, es decir, aquellos bienes o servicios que tienen una relación indirecta con el deporte pero no son necesarios para hacer deporte. Piénsese, por ejemplo, en los hoteles que alojan a deportistas, en los medios de comunicación que retransmiten eventos deportivos, o en las apuestas que se realizan sobre un determinado resultado deportivo. Esta definición ampliada comprende más de 100 tipos de negocios, de acuerdo con la NACE, y sería la aproximación más completa al impacto económico del deporte.

\section{METODOLOGÍA: LAS CUENTAS SATÉLITE}

Además de una definición consensuada de lo que ha de medirse se necesita, obviamente, un acuerdo sobre cómo hacerlo para alcanzar resultados comparables en el seno de la UE. Desde el punto de vista macroeconómico se han utilizado tres instrumentos para medir la importancia económica del deporte (Pedrosa y Salvador, 2003): la tabla económica de conjunto, las cuentas satélite y el análisis input output. Pues bien, desde el primer momento el grupo de trabajo propuso la elaboración de las cuentas satélite del deporte. Esta misma solu- 
ción se había adoptado años atrás para el turismo donde, desde 2000, existe una metodología respaldada por el Eurostat que ha permitido avances significativos en este ámbito a nivel europeo (Eurostat, 2010). De hecho, en el caso del deporte, algunos países como Francia, Alemania, Austria y el Reino Unido habían elaborado cuentas satélite aunque con enfoques distintos.

Las cuentas satélite, en general, giran en tono a los sistemas de cuentas nacionales y se construyen con una metodología similar para medir la importancia económica de una industria o sector (el turismo, la cultura, o el deporte, por ejemplo) que no tiene una correspondencia clara con las clasificaciones estadísticas existentes. De esta manera, se logra presentar la información de una manera coherente, y comparable con otros sectores económicos, sobre cómo se produce y se financia la actividad económica sujeta a estimación.

Según Andreff (2002), las cuentas satélite, igual que la tabla económica de conjunto, permiten conocer, utilizando la contabilidad por partida doble, los equilibrios macroeconómicos básicos $\mathrm{y}$, además, a diferencia de ésta última, admitiría completar la información con magnitudes no monetarias aunque, en la práctica, las limitaciones estadísticas y las hipótesis utilizadas no ofrecen un tratamiento satisfactorio de las transacciones realizadas al margen del mercado. El mismo Andreff señaló que el principal inconveniente de las cuentas satélite se encontraba en los costes, en términos de tiempo de trabajo, que suponía. Otra carencia, apuntada por el mismo autor, es que no ofrece, como hace el análisis input output, una valoración de la incidencia indirecta del deporte a través de los efectos que generarían los consumos intermedios en otras ramas industriales.

La ventaja más evidente de disponer de una definición económica del deporte y de una metodología para su estimación comunes es que podrán obtenerse indicadores comparables sobre sus principales macromagnitudes. Pero no se trata, simplemente, de un esfuerzo destinado al conocimiento estadístico. Se pretende, además, que tenga implicaciones para la política económica. En este sentido, un documento presentado a la reunión de directores generales del deporte de la Unión Europea que se celebró en Barcelona en febrero de 2010 (Meerwaarde y SpEA, 2010) proponía una serie de cuestiones que podrían ser resueltas con los datos contenidos en las cuentas satélite del deporte.

En primer lugar, a través del PIB y del empleo asociado al deporte, se dispondrían de indicadores convencionales que permiten conocer, de una manera rigurosa, el tamaño real del sector. Todos los agentes implicados tendrían una referencia básica para valorar la contribución económica de la actividad deportiva en relación con otros sectores productivos. Sobre este tema, Késenne (2002) advirtió con cierta ironía, cuando analizaba los errores de interpretación de los estudios sobre incidencia económica, que "si se sumaran las cifras de todos los estudios de incidencia macroeconómica de los diferentes sectores e industrias nacionales, el PIB calculado sería probablemente cinco veces mayor 
que el PIB real". Por otro lado, también señaló que la importancia cuantitativa de un sector no debe ser un argumento para justificar las subvenciones públicas como, a veces, parece interpretarse por los órganos que financian alguno de los estudios sobre el impacto económico del deporte. De hecho, el sector deportivo, en términos cuantitativos, no está entre los sectores más importantes y su apoyo público se defiende mucho mejor por los efectos externos positivos (sobre la salud, por ejemplo) que provoca. En consecuencia, aún siendo relevante la cifra que representa el "sector deporte" sobre el PIB, existen otros aspectos más interesantes desde el punto de vista de la política económica que podrían quedar patentes cuando se elaboran las cuentas satélite.

En segundo lugar, podrían identificarse mejor las consecuencias de la expansión de la actividad deportiva sobre el crecimiento económico. Se trataría de constatar si el deporte genera una dinámica que estimula el desarrollo económico. Si conocemos de dónde provienen sus inputs y hacia dónde se destinan sus outputs estaremos en condiciones de apreciar mejor los efectos económicos de una expansión del sector y su grado de integración con el resto de actividades productivas ya sean nacionales, regionales o locales. En este punto, la elaboración de las tablas input output del deporte sería un buen complemento.

En tercer lugar, las cuentas satélite del deporte nos aportarían una valiosa información para la evaluación de las políticas públicas que inciden sobre el sector. Piénsese, por ejemplo, en una alteración de la política fiscal aplicada a las asociaciones deportivas o en un cambio en el régimen de la propiedad de las instalaciones destinadas a la práctica del deporte. Esto, lógicamente, provocaría una modificación en los flujos de renta que podría detectarse sin dificultad a través de las cuentas satélite.

En cuarto lugar, también ayudaría a establecer proyecciones sobre la evolución del mercado de trabajo relacionado con el deporte. Si se acepta la hipótesis de un aumento de la demanda de deporte (en todos los sentidos) entonces la disponibilidad de información relevante sobre aspectos tales como la productividad de las industrias del deporte nos permitiría cuantificar su potencial impacto sobre el empleo.

En quinto lugar, es posible que a través de las cuentas satélite encontremos algún apoyo para estimar la rentabilidad social de las inversiones en instalaciones o eventos deportivos. Este es un tema que, desde el punto de vista de la política económica, es muy complejo (Benítez, 1991). No obstante, es indudable que si se disponen de cuentas que determinen las relaciones económicas y financieras asociadas al deporte se facilitaría en gran medida esa labor.

Por último, la cuantificación de los flujos financieros contenidos en las cuentas satélite posibilita el análisis de las consecuencias de aplicar diferentes políticas respecto a asuntos tales como la regulación de las apuestas deportivas o los efectos de los diferentes modelos de patrocinio en el deporte. 


\section{ESTIMACIONES}

Según Andreff (2006) las primeras cuentas satélite para el deporte que se elaboraron en Europa se realizaron en Francia para el año 1971 (MalenfantDauriac, 1977). Después de este trabajo pionero surgieron otros como el de Weber et alia (1995) para Alemania. Aunque han sido más frecuentes, como en el caso de España, las simples propuestas metodológicas que no llegan a convertirse en una verdadera estimación de las cuentas satélite para el deporte. En este punto, podrían citarse el trabajo de Isla y Otero (2000) para Andalucía y el de García y Segura (2003) para Cataluña. En esta línea cabría mencionar también, para el caso de Colombia, a Ruiz, Argiro y Mesa (2010).

No obstante, los primeros países que iniciaron la elaboraron unas cuentas satélite del deporte, basándose en la "definición Vilna" ampliada y en una metodología común, fueron Austria, Chipre y Reino Unido a los que, posteriormente, se unió Polonia. Sus principales resultados se recogen en la Tabla 2. Estas cifras estiman sólo los efectos directos del deporte, aunque también se calcularon los indirectos (como consecuencia de las compras entre empresas) y los inducidos (causados por los incrementos de renta), junto con otra información complementaria.

Tabla 2

Impacto económico del deporte en algunos países europeos

\begin{tabular}{|l|c|c|c|c|}
\hline \% sobre el total nacional & $\begin{array}{c}\text { Austria } \\
(\mathbf{2 0 0 5 )}\end{array}$ & $\begin{array}{c}\text { Chipre } \\
\mathbf{( 2 0 0 4 )}\end{array}$ & $\begin{array}{c}\text { Polonia } \\
(\mathbf{2 0 0 6 )}\end{array}$ & $\begin{array}{c}\text { Reino Unido } \\
(\mathbf{2 0 0 6 )}\end{array}$ \\
\hline Consumo & 3.6 & 3.7 & 2.1 & 2.9 \\
\hline Empleo & 6.4 & 2.2 & 1.5 & 2.5 \\
\hline VAB & 4.9 & 2.4 & 2.0 & 2.3 \\
\hline
\end{tabular}

Fuente: Education and Culture D.G. (2011).

En el caso de Austria es destacable el elevado porcentaje, en relación con los otros países, que representa el empleo y el VAB relacionado con el deporte: 6.4 y 4.9, respectivamente. En cambio su consumo no es, en términos relativos, tan elevado. Estos datos son compatibles con un país que es exportador neto de deporte ya que buena parte de la producción está destinada a turistas que, en gran medida, provienen de Alemania. De hecho, algo más del $50 \%$ de la producción relacionada con el deporte en Austria se corresponde con hoteles y restaurantes (SIRC, 2011).

Las cifras de Chipre son, en términos absolutos, muy reducidas teniendo en cuenta que su población no supera el millón de habitantes. No obstante, los porcentajes de empleo y producción atribuibles al deporte son parecidos a los que registran países de mayor tamaño como el Reino Unido. En cambio, es el 
país que tiene el mayor porcentaje de consumo relacionado con el deporte respecto al total de los países analizados. De acuerdo con el informe elaborado por el SIRC (2011), más del $60 \%$ del consumo deportivo en Chipre puede atribuirse a las apuestas relacionadas con el deporte, a la gestión de instalaciones deportivas, y a las actividades de los clubes, gimnasios, etc.

En Polonia también quedó demostrada la importancia económica del deporte. Los sectores que más VAB generaron debido al deporte fueron los servicios recreativos y deportivos, la educación, el comercio, los servicios de transporte, manufacturas (industria del metal, equipamiento deportivo, ropa, productos farmacéuticos, básicamente) y construcción. En términos de empleo, las mayores participaciones se dieron en educación y servicios recreativos y deportivos (Liberda, 2011).

Por último, el Reino Unido, igual que ocurre con Austria, presenta un porcentaje de empleo relacionado con el deporte superior al de la producción en el total de la economía. Quizás merezca reseñarse la importancia relativa que adquieren en el Reino Unido las apuestas deportivas (Education and Culture D.G., 2011). Recientemente, se ha publicado un informe que incluye datos para el periodo 2004-06 y que constata el crecimiento de la economía relacionada con el deporte, tanto en términos de producción como de empleo (SIRC, 2011).

\section{LA REFERENCIA ESPAÑOLA}

Aparte de algunos trabajos que contienen ciertas referencias puntuales, como el de García et alia (2009), nos consta la realización de dos estimaciones sobre la importación del deporte en el conjunto de la economía española. La primera es de Alonso et alia (1991) donde se concluyó, con datos de 1990, que el deporte contribuía en $1.2 \%$ al PIB español. La segunda es de Lera, Rapún y Aguirre (2008). En este último caso, se realizó una encuesta telefónica a 640 hogares para conocer, entre otros aspectos, la importancia económica del deporte. La información estaba referida a 2006 y estimó que el deporte suponía el $2.4 \%$ del PIB en España. La comparación de ambos porcentajes llevó a los autores a afirmar que, "con las debidas cautelas", la importancia económica del deporte se había duplicado en España en 16 años. Es decir, parece que una hipótesis plausible es que ha crecido la participación del deporte en el PIB. No obstante, los propios autores reconocieron las limitaciones de sus cálculos aludiendo a que las respuestas recibidas por los encuestados a ciertas preguntas "no son del todo satisfactorias". Asimismo, señalan la escasez de estudios a nivel nacional que impide la contrastación de resultados. Por otro lado, es muy significativo que el trabajo termine apuntando la necesidad de utilizar un marco teórico y una metodología comunes para obtener comparaciones entre países y, además, se mencione expresamente las cuentas satélite sobre las que ya estaban trabajando algunos países europeos. 
A nivel regional, y al margen de las valoraciones concretas que se han realizado sobre determinados eventos deportivos como, por ejemplo, las de Brunet (1994) para los juegos olímpicos de Barcelona o las que Otero (2009) recoge para el caso andaluz, se dispone de algunas aportaciones sobre la incidencia macroeconómica del deporte que se detallan en la Tabla 3.

Tabla 3

Estudios sobre la incidencia económica del deporte en España

\begin{tabular}{|l|l|c|}
\hline Ámbito territorial & \multicolumn{1}{|c|}{ Autores } & PIB (\%) \\
\hline Andalucía & Otero et alia (2001) & 2.6 \\
\hline Andalucía & Instituto de Análisis Económico y Empresarial de Andalucía (2010) & 3.2 \\
\hline Castilla y León & Pedrosa et alia (2000) & 1.4 \\
\hline Cataluña & Consell Català de l'Esport (2010) & 2.3 \\
\hline Guipuzcoa & Arruza et alia (2011) & 1.7 \\
\hline Navarra & Rapún et alia (2008) & 1.8 \\
\hline Pais Vasco & KPGM Consulting (2002) & 1.9 \\
\hline España & Alonso et alia (1991) & 1.2 \\
\hline España & Lera, Rapún y Aguirre (2008) & 2.4 \\
\hline
\end{tabular}

Fuente: Elaboración propia.

Es muy significativo que los estudios sobre la incidencia económica del deporte en España hayan sido financiados por instituciones públicas relacionadas con el deporte lo que nos hace recordar las palabras de Kesénne (2002): “ ... a la mayoría de ellos [los economistas] nos les gustan los estudios sobre incidencia económica, se diría que sólo les gustan a los políticos y a los miembros de los lobbies del deporte ... con demasiada frecuencia los resultados se utilizan para justificar que el sector deportivo debe recibir más subvenciones". No obstante, debe recordarse que sin la aportación de fondos públicos difícilmente podrían acometerse determinadas investigaciones. En cualquier caso, con independencia de la utilización interesada que se realice de estos estudios, parece necesario que, en el contexto de la Unión Europea, España realice un esfuerzo por construir las cuentas satélites del deporte y, en este sentido, queda un buen trecho por recorrer.

En España existen algunas fuentes estadísticas muy valiosas para el estudio del impacto económico del deporte. Por el lado de la oferta, el Censo Nacional de Instalaciones Deportivas 2005 (Consejo Superior de Deportes, 2007), cuya realización fue financiada conjuntamente por el Consejo Superior de Deportes y las Consejerías competentes de cada Comunidad Autónoma, tienen un elevado grado de desagregación y son un poderoso instrumento no sólo para la planificación sino para el conocimiento del tipo de infraestructuras destinada a la práctica deportiva. Por cierto que en la publicación que acabamos de mencionar 
se puede leer en diferentes ocasiones que el sector deportivo supone el $1.6 \%$ del PIB, y se cita como referencia a Arévalo (2006), "lo que comienza a ser uno de los sectores económicos con mayor potencial del país" (sic). Pues bien, en el trabajo de Arévalo el porcentaje que se recoge es el aportado por Alonso et alia (1991) que, como se ha mencionado más arriba, es el 1,2\% referido a principios de la década de los 90. Al margen de este desliz, en el censo se detecta un sustancial incremento de las instalaciones deportivas en España. En 1986, cuando se realizó el primer censo, existían 48.723. En el segundo censo, editado en 1998, aunque el trabajo de campo se efectuó entre octubre de 1996 y abril de 1997, se registraron 66.670. Y en el último, referido a 2005, se contabilizaron 79.059. Los datos, en consecuencia, parecen ser compatibles con la hipótesis de que, por el lado de la oferta, atendiendo a la definición estadística de Vilna (la más restrictiva y que se corresponde con la categoría 92.6 de la NACE Rev. 1), se ha producido un aumento en España de la importancia económica del deporte.

Por otro lado, por el lado de la demanda se dispone, desde 1980, de una encuesta quinquenal sobre los hábitos deportivos de los españoles realizada por el Consejo de Investigaciones Sociológicas por encargo del Consejo Superior de Deporte. La última disponible está referida 2010 (García y Llopis, 2011). Comparando los resultados observamos que mientras que en 1980 el porcentaje de población, mayor de 15 años, que practicaba uno o varios deportes era del $22 \%$, en 2010 fue del $40 \%$. Además, mientras que en 1980 la mitad de los encuestados declararon estar poco o nada interesados en el deporte, en 2010 descendió al $37 \%$. Es decir, esos datos podrían sostener la tesis de que tanto el gasto en deporte activo, vinculado a la práctica deportiva, como el pasivo, asociado al disfrute del deporte como espectáculo, han aumentado en el periodo considerado o, dicho de otra manera, que ha crecido la importancia económica del deporte.

No obstante, al margen de aproximaciones parciales, no se dispone de una información completa que permita la construcción de las cuentas satélite del deporte en España. En efecto, aunque, tal como se ha señalado, se han realizado aportaciones destacables que estiman el PIB del deporte a nivel nacional o de algunas regiones, un análisis riguroso exigiría, al menos, conocer, en primer término, los distintos componentes que integran la demanda agregada: consumo, inversión, gasto público y exportaciones netas.

\subsection{Consumo}

Habría de estimarse el gasto de los hogares en bienes y servicios relacionados con el deporte: ropa, calzado, prensa, apuestas, entradas, cuotas, equipo, transporte, etc. La Encuesta de Presupuestos Familiares elaborada por el INE permite conocer la evolución de algunas partidas. En concreto, se dispone de información del gasto total de los hogares de acuerdo con la clasificación de 
bienes y servicios COICOP (Classification of Individual Consumption According to Purpose) a 5 dígitos lo que facilita una aproximación al consumo privado en deporte. Por ejemplo, existen datos sobre el gasto en grandes equipos relacionados con los deportes y el ocio al aire libre (código 09211) y en lugares cubiertos (09222), en equipo para el deporte, camping y entretenimiento al aire libre y sus reparaciones (09321), y en servicios recreativos y deportivos (09411). Por otro lado, las memorias elaboradas por el Ministerio de Economía y Hacienda proporcionan información estadística relevante sobre las apuestas deportivas.

Las mencionadas fuentes estadísticas posibilitan una aproximación al consumo agregado relacionado con el deporte tal como recoge la Tabla 4 para el año 2010. De entrada, advertimos que la cifra de consumo (4.824 millones de euros) está claramente infravalorada ya que si bien es cierto que se incluyen, ya que no se disponen de datos desagregados, algunos gastos más relacionados con el ocio que con el deporte (como las caravanas, por ejemplo) no se ha podido incluir, por la misma razón, otros gastos como los destinados a ropa y calzado deportivo que, según los cálculos realizados por Rapún et alia (2008), supusieron algo más del $20 \%$ del consumo en deporte en España en el año 2006. De hecho, estos autores estimaron que el consumo en deporte representaba el 3,5\% del total nacional. Por nuestra parte, tomando los datos de la Contabilidad Nacional del INE, los 4.824 millones de euros que incluyen las partidas señaladas sólo representan el $0,8 \%$ del gasto en consumo final de los hogares.

Lógicamente, los datos anteriores son insuficientes y, en consecuencia, es preciso el diseño y la realización de una encuesta para conocer todos los componentes del consumo de las hogares en deporte y, sobre esa base, llegar a una cifra agregada.

\subsection{Inversión}

Se trataría de estimar la formación bruta de capital realizada por el sector privado, es decir, tanto instituciones sin fines de lucro (federaciones, clubes, asociaciones) como empresas o particulares que realicen inversiones relacionadas con el deporte. Aunque podría discutirse la pertinencia de incluir determinadas partidas (como, por ejemplo, los recursos dedicados por los clubes profesionales al entrenamiento y la formación de futbolistas) la principal aportación de estas entidades se refiere a la inversión en instalaciones deportivas. En este caso, existen algunas fuentes estadísticas de cierta utilidad como el Registro de Actividades Económicas, el Registro Mercantil o la Encuesta Anual de Servicios del INE. En este último caso, encontramos una estimación de la inversión en activos materiales de las "actividades deportivas, recreativas y de entretenimiento" que se corresponde con los códigos 931 y 932 de la CNAE-2009. Esta 
partida supuso 802 millones de euros en 2009. En términos estrictos esta partida supera la definición más restringida de Vilna ya que habría de incluir sólo las actividades del código 931 de la CNAE y no, tal como recoge el dato, añadirle las del código 932. Una vez más, la ausencia de información impide afinar el dato. Aunque, de entrada, podría suponerse que se está sobreestimando, por esta razón, la inversión relacionada con el deporte debemos señalar que, por otro lado, se están ignorando las inversiones de otras empresas (como, por ejemplo, las que fabrican productos necesarios para la práctica deportiva). En consecuencia, también es evidente que una estimación rigurosa conllevaría la realización de encuestas específicas.

\subsection{Gasto Público}

La construcción de una cuenta para el sector público obligaría a cuantificar las distintas fuentes de financiación y los gastos correspondientes. Es obvio, que los presupuestos de las distintas administraciones públicas a todos los niveles aportan datos muy útiles. Por ejemplo, los Presupuestos Generales del Estado de 2010 contienen tres programas directamente vinculados con el deporte: cooperación, promoción y difusión cultural en el exterior, en el área deportiva (programa 144A); deporte en edad escolar y en la universidad (programa $322 \mathrm{~K}$ ); y fomento y apoyo de las actividades deportivas (programa 336A). La suma de estos programas alcanzó los 181 millones de euros que, como es obvio, debe considerarse como un indicador mínimo del gasto público real en deporte. En cualquier caso, la recogida de información precisa de ingresos y de gastos de este sector institucional obligaría a realizar encuestas específicas.

\subsection{Exportaciones netas}

Para completar el cuadro macroeconómico por el lado de la demanda necesitaríamos las cifras correspondientes a las importaciones y exportaciones del sector deportivo. De entrada, la base de datos Estacom del Instituto Español de Comercio Exterior (ICEX) del Ministerio de Economía y Competitividad proporciona una información muy completa sobre algunos artículos relacionados con el deporte. Así se facilitan datos sobre las importaciones y exportaciones de artículos deportivos (que incluyen, entre otros, el calzado y la ropa deportiva), de armas deportivas y municiones, y de la náutica deportiva.

Como se observa en la Tabla 4, la mayor parte del déficit comercial se explica por la partida "náutica deportiva" donde se cuantifican, no sólo las importaciones y exportaciones de embarcaciones propiamente deportivas, sino también las recreativas. Teniendo en cuenta, además, que no se disponen de datos sobre el comercio exterior de servicios donde podría manejarse la hipótesis de que España es un exportador neto, dada la llegada de turistas extranjeros cuya principal motivación es la práctica deportiva (el caso del golf puede ser un 
buen ejemplo), cabe afirmar que la cifra de 1.741 millones de euros está sobrevalorando el déficit exterior español en el sector deportivo.

\subsection{Aproximación tentativa a la importancia económica del deporte}

Por todo lo expuesto podría afirmarse que los 4.066 millones de euros que suman las partidas anteriormente señaladas, y que contiene la Tabla 4, están infravalorando la dimensión real del sector deportivo en España. Esa cifra representa, tomando como referencia la contabilidad nacional del INE, el 0,4 \% del PIB. Este porcentaje, dado los estudios previos existentes, nos confirma que, por un lado, con la información estadística disponible solo puede estimarse, de manera muy imperfecta, la importancia económica del deporte en España y, por otro lado, que es necesario un esfuerzo adicional, que conlleva la realización de encuestas ad hoc para poder construir las cuentas satélite del deporte en la misma línea que se está desarrollando en otros países europeos.

Tabla 4

Indicadores de la demanda agregada relacionada con el deporte. 2010

\begin{tabular}{|l|c|}
\hline \multicolumn{1}{|c|}{ MACROMAGNITUD } & Millones de $€$ \\
\hline \multicolumn{1}{|c|}{ CONSUMO } & 4.824 \\
\hline Gastos en apuestas deportivas & 509 \\
\hline Grandes equipos relacionados con los deportes y el ocio al aire libre & 451 \\
\hline Grandes equipos relacionados con los deportes y el ocio en lugares cubiertos & 73 \\
\hline Equipos para el deporte, camping y entretenimiento al aire libre y sus reparaciones & 622 \\
\hline Servicios recreativos y deportivos INVERSIÓN & 3.169 \\
\hline & 802 \\
\hline Inversión en activos materiales de las actividades deportivas, recreativas y de & 802 \\
\hline entretenimiento (dato referido a 2009) & 181 \\
\hline \multicolumn{1}{|c|}{ GASTO PÚBLICO } & 3 \\
\hline Cooperación, promoción y difusión cultural en el exterior & 19 \\
\hline Deporte en edad escolar y en la Universidad & 159 \\
\hline Fomento y apoyo de actividades deportivas & -1.741 \\
\hline \multicolumn{1}{|c|}{ EXPORTACIONES NETAS } & -621 \\
\hline Artículos deportivos & 85 \\
\hline Armas deportivas y municiones & \\
\hline Náutica deportiva & -1.205 \\
\hline & 4.066 \\
\hline
\end{tabular}

Fuente: INE y Ministerio de Economía y Hacienda. Elaboración propia.

\section{CONCLUSIONES}

Los intentos de valorar la importancia económica del deporte en Europa se iniciaron a finales de la década de los ochenta y se ocuparon básicamente de 
cuantificar su participación en el PIB. No obstante, la ausencia de una metodología común limitaba la posibilidad de disponer de datos fiables y comparables sobre el peso económico del deporte. Sólo muy recientemente, y gracias al impulso de las instituciones europeas, se han producido avances significativos en este ámbito. En particular, el grupo de trabajo sobre economía y deporte, auspiciado por la Comisión Europea, ha planteado una propuesta sobre la definición del deporte y sobre la utilización de una misma metodología que está permitiendo el conocimiento, sobre unas bases rigurosas, del impacto económico del deporte y que, inicialmente, se ha aplicado a Austria, Chipre, Polonia y Reino Unido.

Los primeros resultados confirman la hipótesis de que existe una amplia diversidad de situaciones en el seno de Unión Europea aunque se advierte que el deporte representa porcentajes significativos del consumo, del empleo y del $\mathrm{VAB}$. Es en términos de empleo donde se registra la mayor diferencia de resultados al variar del $6.4 \%$ de Austria hasta el $1.5 \%$ de Polonia. Ahora bien, aun siendo interesante la cuantificación, con criterios homogéneos, de la importancia relativa que el deporte puede tener en las principales macromagnitudes mucho más relevante, desde el punto de vista de la política económica, es la construcción de las cuentas satélite del deporte tal como se contempla en el proyecto europeo. En efecto, cuando se disponga de una información coherente sobre cómo se financian y se producen las actividades relacionadas con el deporte sabremos, entre otras cosas, no sólo el tamaño real del sector sino también podrían identificarse las consecuencias de su expansión sobre el crecimiento y el mercado de trabajo. Así mismo, se habrá conseguido un valioso instrumento para la evaluación de las políticas públicas aplicadas al deporte.

En el caso particular de España se han llevado a cabo algunos estudios, tanto a nivel nacional como regional, sobre la incidencia económica del deporte que han sido financiados por instituciones públicas relacionadas con el sector y que, la mayoría de ellos, se realizaron a partir del año 2000. No obstante, parece inevitable que también España acabe construyendo, en el contexto europeo, sus propias cuentas satélite. En este sentido, existen indicios que demuestran que, tanto por el lado de la demanda como de la oferta, ha crecido la importancia económica del deporte en las últimas décadas.

Por último, con las estadísticas disponibles hemos construido determinados indicadores de los distintos componentes que integran la demanda agregada. A partir de ahí se ha realizado una estimación tentativa de la importancia relativa del deporte en el PIB. En este contexto, tenemos razones suficientes para pensar que el $0.4 \%$ que se ha obtenido infravalora la verdadera dimensión que representa el deporte en el PIB español y, en cualquier caso, demuestran la necesidad de realizar encuestas adicionales para disponer de la información que se precisa para elaborar las cuentas satélite. 


\section{REFERENCIAS BIBLIOGRÁFICAS}

AHLERT, G. (2000): "Reasons for Modeling Sports in a Complex Economic Model: Two Examples", European Journal for Sport Management, 1, pp. 3155.

ALONSO, J. et alia (1991): "Impacto Económico del Deporte en España." Revista de Investigación y Documentación sobre las Ciencias de la Educación Física, 18, pp. 23-35.

ANDREFF, W. (2002): "Los intentos europeos y franceses de elaborar una contabilidad nacional de la economía del deporte". En OTERO (2002), pp. 23-53.

ANDREFF, W. (2006): "Sports accounting". En ANDREFF y SZYMANSKI (2006), pp. 11-21.

ANDREFF, W. et alia (1994): The Economic Impact of Sport in Europe: Financing and Economic Impact, Background Document, $14^{\text {th }}$ Informal Meeting of European Sports Ministers, Strasbourg, Council of Europe, 28-29 April.

ANDREFF, W. y SZYMANSKI, S. (2006): Handbook of the Economics of Sport, Cheltenham, Northampton, Edward Elgar.

ARÉVALO, M. (2006): Las Fundaciones Deportivas Españolas, Tesis Doctoral, Universidad de Alcalá.

ARRUZA, J. et alia (2011): Estudio de la Actividad Económica del Deporte en Gipuzkoa, Observatorio del Deporte de Gipuzkoa.

BEDNARIK, J., KOLAR, E. y JURAK, G. (2010): "Analysis of the sports services in Slovenia", Kinesiology, 42, pp.142-152

BENÍTEZ, J.J. (1991): "El análisis coste beneficio como técnica al servicio de la política económica", Hacienda Pública Española, 117, pp. 43-56.

BRUNET, F. (1994): Economía de los Juegos Olímpicos de Barcelona 1992, Lausana.

COMISIÓN DE LAS COMUNICADES EUROPEAS (2007): Libro Blanco sobre el Deporte, Bruselas

CONSEJO SUPERIOR DE DEPORTES (2007): Censo Nacional de Instalaciones Deportivas. 2005, Madrid, 2007.

CONSELL CATALÀ DE L'ESPORT (2010): El Pes Econòmic de l'Esport a Catalunya, Consell Català de l'Esport, Esplugues de Llobregat.

DIMITROV, D., et alia (2006): Die makroökonomischen Effekte des Sports, Europa, Studie im Auftrag des Bundeskanzleramts, Sektion Sport, Wien, 2006.

EDUCATION AND CULTURE D.G. (2011): Sport Satellite Accounts. A European Project: New Results.

EUROSTAT (2010): Tourism Satellite Accounts (TSA) in Europe. 
GARCÍA, M.I. et alia (2009): “La aportación económica de la industria de la cultura y el ocio en España", Estudios de Economía Aplicada, 27, pp.61-86.

GARCÍA, M. y LLOPIS, R. (2011): Ideal Democrático y Bienestar Personal. Encuesta sobre Hábitos Deportivos en España 2010, Consejo Superior de Deportes y Centro de Investigaciones Sociológicas, Madrid.

GARCÍA, J. y SEGURA, M. (2003) : Comptabilitat Satèl-lit de l'Esport a Catalunya. Institut d'Estatistica de Catalunya.

INSTITUTO DE ANÁLISIS ECONÓMICO Y EMPRESARIAL DE ANDALUCÍA (2010): Actividad Económica del Deporte en Andalucía, 2008.

ISLA, F. y OTERO, J.M. (2002): "Disaggregation the economic flows of sports in the Andalusian Input-Output framework", The International Association of Sports Economics, $4^{\text {th }}$ Annual Conference, Columbia University, Nueva York.

JONES, H. (1989): The Economic Impact and Importance of Sport: An European Study, Council of Europe, Strasbourg.

KESÉNNE, S. (2002): "El problema de los estudios de incidencia económica del deporte”. En Otero (2002), pp. 91-99.

KPGM Consulting (2002), Kirolari loturiko jarduerek Euskadiko ekonomian duten eragma neurtzeko azterlana, Euskadi Kirola.

LERA, F., RAPÚN M. y AGUIRRE, J.: (2008): Análisis y Evaluación Económica de la Participación Deportiva en España, Consejo Superior de Deportes, Madrid.

LIBERDA, Z.B. (2011): Sport Satellite Account for Poland, $58^{\text {th }}$ ISIS World Statistics Congress, Dublin.

MALENFANT-DAURIAC, C. (1977): L'Economie du Sport en France. Un compte Satellite du Sport, Cujas, Paris.

MEEWAARDE y SpEA (2010): The Use of Sport Satellite Accounts for Policy Purpose, http://www.esce.at/spea/speafile/100226\%20SportSatelliteAccounts\%20Poli cy\%20Purposes\%20Meerwaarde\%20SpEA\%20EN.pdf

MINISTĖRE DE LA SANTÉ ET DES SPORTS (2009): “Le poids économique du sport en 2007", Bulletin de statistiques et d'études, Septembre.

MUSSINO, A. (1994): "The Sport System in Italy, Statistical Tools for the Analysis of Supply and Demand", Improving Evaluation of Sports through Statistics, CONI, Roma.

NOLL, R. G.: "Sports Economics after fifty years". En RODRÍGUEZ, KÉSENNE y GARCIA (2006), pp. 17-49.

OTERO, J.M. (2009): El impacto socioeconómico del deporte: métodos de análisis y evaluación, Seminario XREAP, Facultad de Economía y Empresa, Universidad de Barcelona.

OTERO, J.M. et alia (2001): Estudio Socioeconómico del Deporte en Andalucía 1998-1999, Junta de Andalucía, Consejería de Turismo y Deporte. 
OTERO, J.M. (coord.) (2002): Incidencia Económica del Deporte, Instituto Andaluz del Deporte.

PEDROSA, R. et alia (2000): El impacto económico del deporte en Castilla y León, Junta de Castilla y León, Consejería de Educación y Cultura.

PEDROSA, R. y J.A. SALVADOR (2003): "El impacto del deporte en la economía: problemas de medición", Revista Asturiana de Economía, 26, pp. 6184.

RAPÚN, M. et alia (2008): "Impacto del deporte en la economía navarra", Cuadernos Técnicos de Deporte, 14. Pamplona, Gobierno de Navarra

RODRÍGUEZ, J., KÉSENNE, S. y GARCÍA, J. (2006): Sports Economics after Fifty Years: Essays in Honour of Simon Rottenberg, Servicio de Publicaciones de la Universidad de Oviedo

ROTTENBERG, S. (1956): “The Baseball Players' Labor Market”, Journal of Political Economy, 64, pp. 242-58.

RUIZ, A., ARGIRO, E. Y MESA, R.J. (2010): "Medición económica del deporte en Colombia: una propuesta metodológica de cuenta satélite", Lecturas de Economía, 72, pp. 141-167,

SOEK, J. (2006): "Sport in National Sports Acts and Constitutions: Definition, Ratio and Purposes", The Internacioal Sports Law Journal, 3-4, pp. 28-35

SIRC (2010): Economic Value of Sport in England. 1985-2008. Sheffield Hallam University

SITC (2011): 2004-2006 Sport Satellite Account for the UK, Sheffield Hallam University

TASK, M. y S. KÉSENNE (2000): "The Economic Significance of Sport in Flanders", Journal of Sport Management, 4, pp. 342-365.

VOCASPORT (2004): Vocational Education in Training in the Field of Sport in the European Union: Situation, Trends and Outlook, http://www.kirolan.org/El\%20sector\%20del\%20empleo\%20deportivo/0.1.1\% 20lotvocasport_en.pdf.

WEBER, W. et alia (1995): Die wirtschaftliche Bedeutung des Sports, Schorndorf, Hofmann. 